TITLE:

\title{
Manipulation of Single Atoms by Atomic Force Microscopy as a Resonance Effect
}

\section{AUTHOR(S):}

Kim, Byungsoo; Putkaradze, Vakhtang; Hikihara, Takashi

\section{CITATION:}

Kim, Byungsoo ... [et al]. Manipulation of Single Atoms by Atomic Force Microscopy as a Resonance Effect. Physical Review Letters 2009, 102(21): 215502.

ISSUE DATE:

2009-05

URL:

http://hdl.handle.net/2433/109868

RIGHT:

(c) 2009 The American Physical Society 


\title{
Manipulation of Single Atoms by Atomic Force Microscopy as a Resonance Effect
}

\author{
Byungsoo Kim* and Vakhtang Putkaradze \\ Department of Mathematics, Colorado State University, Fort Collins, Colorado 80235, USA
}

Takashi Hikihara

Department of Electrical Engineering, Kyoto University, Katsura, Nishikyo, Kyoto, 615-8510 Japan

(Received 22 March 2009; published 27 May 2009)

\begin{abstract}
Extraction and deposition of single atoms using an atomic force microscope tip is a promising technique for building nanostructures. Previous theoretical models for this technique, based on adiabatic atom motion in either classical or quantum mechanics settings, encountered an apparent difficulty in explaining atom extraction and deposition for the same experimental conditions. We resolve that difficulty by showing that both extraction and deposition of atoms can be formulated in terms of pure classical mechanics as a resonance effect, arising from a combination of interatomic forces and vibrations of individual atoms.
\end{abstract}

DOI: 10.1103/PhysRevLett.102.215502

As electronic devices miniaturize, and the methods for small-scale manufacturing advance, the possibility of building useful structures out of individual atoms is becoming within our reach [1]. The techniques of manipulation of individual atoms have experienced an explosive development in the last two decades. Already, scanning tunneling microscopy has been used for atomic-scale imaging and manipulations [2-5]. However, scanning tunneling microscopy operation is limited to electrically insulating surfaces. In contrast, an atomic force microscope (AFM) uses short range interatomic forces to directly probe and image material surfaces [6], so the tip of the device can be used to manipulate individual atoms for a wide range of materials [7-9]. Recent experiments have demonstrated the possibility of the following manipulations of atoms using an AFM tip. An "adatom," a single atom embedded at the surface in a crystalline lattice, can be extracted by an AFM tip without damaging the surface structure of surrounding atoms [9]. The extracted atom can be deposited back into the defect hole which is created by the previous atom extraction [9]. A single atom embedded in a surface lattice can furthermore be rolled over to the top of the surface and can be dragged laterally by the AFM tip [8]. A defect atom embedded in a surface lattice can be interchanged in its lattice position with a neighboring atom [10]. An atom from the AFM tip can interchange with a surface adatom in a reversible exchange procedure [11]. There have been two major directions for theoretical explanations of these results.

The first avenue of reasoning [5,12] centers on the consideration of a double-well potential model within the framework of classical mechanics. When the AFM tip is far away from the lattice, there are two minima of potential energy for the target atom, one close to the tip and another one close to the surface. These potential minima are separated by a large energy barrier that prevents the target atom from reaching the tip. When the AFM tip is within several
PACS numbers: 62.25. $-\mathrm{g}, 34.20 . \mathrm{Cf}, 46.40 . \mathrm{Ff}, 81.16 . \mathrm{Ta}$

angströms ( $\mathrm{A})$ from the surface, the potential barrier between the two minima disappears, affording the capture by the tip; upon tip withdrawal, the potential barrier reappears and the target atom follows the tip. Since the potential well is asymmetric, this line of reasoning leads to the conclusion that there must be a strong preference towards extraction versus deposition of target atoms. Later in this Letter, we show that it is not necessary for the potential well to disappear completely, and both extraction and deposition may happen even when a finite potential barrier exists, as illustrated on Fig. 3 and the discussion focusing on the energy balance. We shall note, however, that the doublewell structure of the potential plays an important role in our considerations, eventually explaining the asymmetry between target atom behavior for deposition and extraction.

Quantum mechanics offers an alternative to the doublewell model by allowing the target atom to escape even when the potential barrier is higher than the particle's energy. This is due to the phenomenon of tunneling (i.e., particle crossing the classically forbidden zone) as well as possible changes in the barrier because of the covalent bonding structure. Thus, the second avenue of reasoning appealed to the quantum mechanical explanations $[13,14]$. Under this paradigm, the Car-Parrinello molecular dynamics model was used to compute the electronic structure for a given configuration of the tip and the lattice. Then, the target atom was moved to find the minimal energy state according to the electronic density, and the calculation was repeated again. This quantum mechanical model reproduces some aspects of atomic manipulations along a crystal surface. However, analogous to the classical models described above, these quantum mechanical theories did not consider the time scales of atomic vibration and AFM tip. In other words, these quantum mechanical models assumed the experiment is conducted at absolute zero temperature [13]. However, laboratory evidence at nonzero temperature demonstrates that proper excitation of atomic vibrations 
has the ability to break chemical bonds [15], so the vibrations of individual atoms may alter the results of CarParrinello molecular dynamics considerably.

The most interesting to us is the failure of these theories to explain both deposition and extraction of the target atom for the same experimental conditions. This is a consequence of considering the atomic manipulation as the force balance only. Indeed, deposition would require a stronger bond between the lattice and the target as compared to the tip-target bond. On the other hand, atom extraction would require a stronger tip-target bond as compared to the targetlattice bond. Seen from the point of view of forces only, this creates a paradox precluding the possibility for both deposition and extraction of atoms, which is in apparent contradiction with the experiments [9].

We show herein that experimental results pertaining to the extraction and deposition can be explained using a purely classical description based on the simple and familiar idea of parametric resonance [16]. Here, by parametric resonance we mean transfer of energy from tip to target atom through a periodic change of a parameter of the system, in our case, the length between these atoms. The idea of parametric resonance-based microscopy has been presented before; see [17]. In our case, we consider parametric resonance as applied to the motion of individual atomic vibrations and not the whole cantilever. In other words, we demonstrate that the coupled dynamics of the AFM tip, target atom, and surrounding lattice, are capable of producing excitations which extract or deposit the atoms. The novelty of our approach, compared to the previous literature, lies in the consideration of vibrations for individual atoms. Our explanation proceeds as follows.

A typical time scale of individual atomic vibrations is of the order of $10^{13} \mathrm{~Hz}$ [18]. The AFM tip itself is an assembly of atomic oscillators-silicon (Si) atoms in experiments-of approximately the same frequency. The dynamics of AFM cantilever oscillation is much slower than the atomic vibrations: the frequency of oscillations of the AFM tip lies in the range of $10^{3} \mathrm{~Hz}$. Thus, during one AFM tip oscillation, each individual atom performs $\sim 10^{8}$ vibrations. When the AFM tip approaches to the target to about $2 \AA$, the collective dynamics between the tip and the target ensues due to the arising interatomic force between the tip and the target. When the tip stays in near contact with a target atom on the surface, vibration of the atom in the tip works as a periodic forcing on the target atom and thus amplifies the vibration of the target atom. This interaction is a purely classical mechanical effect of parametric resonance. This resonance provides enough energy for a target atom to cross the potential barrier and escape to either the lattice (deposition) or the tip (extraction), without any necessity to either wait for the potential barrier to vanish, or use quantum mechanical tunneling. Also, this scenario allows one to explain atomic extraction and deposition using the same physical mechanism.

To confirm this scenario, we conducted numerical simulations of a simplified 3D model for the dynamics of a single atom under the periodic approach of the AFM tip. The setup for our simulations is illustrated in Fig. 1.

The AFM tip is represented by five $\mathrm{Si}$ atoms in a pyramid configuration with four atoms forming the base on Fig. 1. In reality, the Si tip of the AFM will be covered by an oxidized layer [19]. In experiments [20], special care has been taken to remove the oxidization layer from the AFM tip and performing experiments in ultrahigh vacuum. Unfortunately, the exact degree of deoxidization achieved in experiments is not known. In our theory (see below), oxidization will change the atom geometry, oscillation frequency, as well as the Lennard-Jones interactions. All of these factors should impede the manipulation process. The lattice is modeled by 71 atoms in a proper crystal formation at $Z \simeq 0$. The target atom, which is identical to lattice atoms, is initially positioned in the place of one of the lattice atoms. Numerical simulation proceeds by computing the dynamics of the tip and lattice atoms based on a regular Newtonian mechanics: mass $\times$ acceleration $=$ net force for each atom. For simplicity, the AFM tip atoms are modeled by oscillators of fixed frequency, $5 \times$ $10^{12} \mathrm{~Hz}$, and a fixed amplitude, $0.1 \AA$, at prescribed time-dependent positions. This amplitude corresponds to the temperature of $80 \mathrm{~K}$ per oscillation mode as in experiments. It is also a typical value of atomic vibration amplitude reported earlier [21]. The behavior of the target atom is then a result of many-body dynamics due to atomic interactions.

In this Letter, we shall explain experimental results on atom extraction and deposition at low temperature ( $80 \mathrm{~K})$ [9]. Our model assumes only the classical interatomic pair potential in the Lennard-Jones (LJ) form:

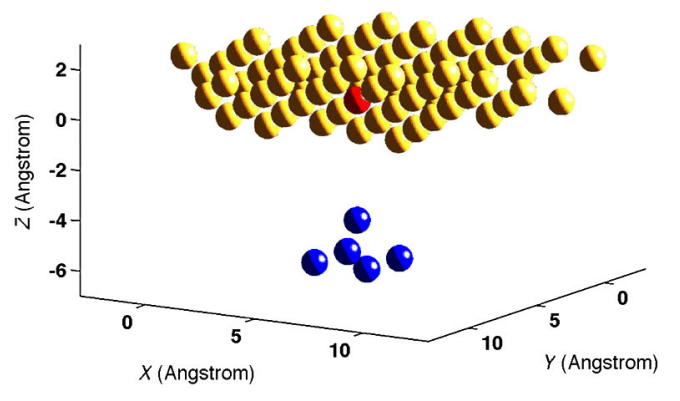

FIG. 1 (color online). Initial arrangement atoms for simulations. Lattice atoms are at $Z \sim 0-2 \AA$ on top with light (yellow) color, the target atom embedded in the lattice is marked with a dark (red) color in the center of the lattice, and AFM tip atoms are set in a tetrahedral structure at $Z \sim-5 \AA$ and marked with dark (blue) color. The tip atoms are considered as fast $5 \times$ $10^{12} \mathrm{~Hz}$ oscillators with fixed amplitude. To model the oscillations of the AFM tip itself, we also prescribe the motion of the tip atoms, bringing them closer to surface and taking them away from the surface with much slower frequency $\sim 10^{10} \mathrm{~Hz}$. The dynamics of all 72 target and lattice atoms is then computed according to the rules of Newtonian mechanics, taking into account all the interatomic forces. 


$$
V_{\mathrm{LJ}}=4 \epsilon\left[\left(\frac{\sigma}{r}\right)^{12}-\left(\frac{\sigma}{r}\right)^{6}\right]
$$

which is similar to the interatomic potential observed in the experiments $[6,9]$. The simulation parameters for the $\mathrm{LJ}$ potential are $\sigma=2 \AA, \epsilon=3.47 \times 10^{-19} \mathrm{~J}(\sim 2.17 \mathrm{eV})$, where $\epsilon$ is the minimum energy for the pair potential. In the experiments, the target atom is surrounded by the atoms of the crystalline lattice, with the average distance between two lattice atoms being $2 \AA$, corresponding to a typical value for a real silicon crystal. All pair potentials are adjusted to conform to the experimentally measured values of interaction and binding energy in the lattice. The binding energy between two atoms is about $-2.5 \mathrm{eV}$ and the binding energy of an atom which is embedded in a bulk lattice is about $-4 \sim-5 \mathrm{eV}$ with a distance of $2 \AA$ between the atoms [18]. Also, in our simulations, all the atoms are assumed to have the mass of a silicon atom, i.e., $4.648 \times 10^{-26} \mathrm{~kg}$.

In general, other potentials may be used in simulations as well. The important feature for our scenario to be valid is: (a) similar frequencies of atomic vibrations for all atoms (tip, target, and lattice), and (b) the relatively short range of the potential so parametric resonance occurs when the AFM tip comes close to the lattice. A reasonably accurate approximation of covalent bonds by potential could be possible, although it would require use of more algebraically complex and direction-dependent interaction potentials. We believe that as long as the potential used for modeling of covalent bonds is short range and does not alter atomic vibration frequency significantly, it will lead to similar parametric excitation of atoms. In the absence of good analytical formulas for approximation covalent bonds as classical potential, we shall leave these studies to the future. Our goal here is to demonstrate that even the simplest LJ potential can explain atomic manipulations.

The oscillation of a real-life AFM tip is a periodic function with amplitude $\sim 10 \mathrm{~nm}$ and frequency $10^{5}-10^{6} \mathrm{~Hz}$. In the simulations, we take a tip performing harmonic oscillations with the same amplitude, but increase the frequency to $10^{10} \mathrm{~Hz}$ in order to speed up the computations. This is necessary because we need to resolve the individual atomic vibrations that have a typical frequency of $10^{13} \mathrm{~Hz}$. One AFM oscillation in our simulation thus contains about $10^{3}$ atomic vibrations. The LJ potential is effectively felt when the tip atom and the target atom are about 2-3 $\AA$ apart. During this time, in our simulations, about 300 atomic vibrations happen, as compared to $10^{7}-10^{8}$ atomic vibrations during contact in the experiment. Unfortunately, resolving a realistic number of atomic oscillations for the complex system we consider is out of reach for modern computers. For real systems, the number of atomic vibrations in contact will increase the energy being transferred from the tip to target atom. Thus, we expect that atomic manipulation in a corresponding experiment will be easier to achieve. On the other hand, even in this reduced setting we are able to demonstrate target atom extraction and deposition.

Figure 2, left panel, shows the distance of the target atom measured normally to the surface as a function of time. One can see that when the AFM tip approaches the target atom, a radical increase of the kinetic energy happens, allowing the target atom to cross the potential barrier and escape the lattice to join the tip atoms. A side view of the target atom extraction is shown in Fig. 2, right panel. Note the large meanderings of the target atom under the extraction process, and the relatively small oscillations of the lattice atoms.

In Fig. 3, we illustrate the energy balance of the system. The left part of Fig. 3 shows the double-well structure of the potential for different distances of the AFM tip apex to the lattice surface. The right part of Fig. 3 compares the kinetic energy of the target atom and the values of the potential barrier of the tip side (dashed) and lattice side (solid black). Initially, the kinetic energy of the target atom is much smaller than the depth of the potential well, but upon the approach of the tip, the kinetic energy quickly increases and at the same time the potential barrier of the double-well potential gets smaller. The target atom escapes to the potential well of the tip and is extracted. The drastic increase of the kinetic energy of the target atom after extraction comes from the continued pumping of energy from the tip atoms. In reality, dissipation in the system due to imperfect vacuum and formation of covalent bonds will dampen those vibrations. Here, we shall neglect the dissipation completely as theoretical modeling for atomic dissipation in this setting is not well established.

Our models demonstrate the deposition procedure simply by replacing the apex atom of the AFM tip by the target atom. The dynamical process of the deposition is illus-
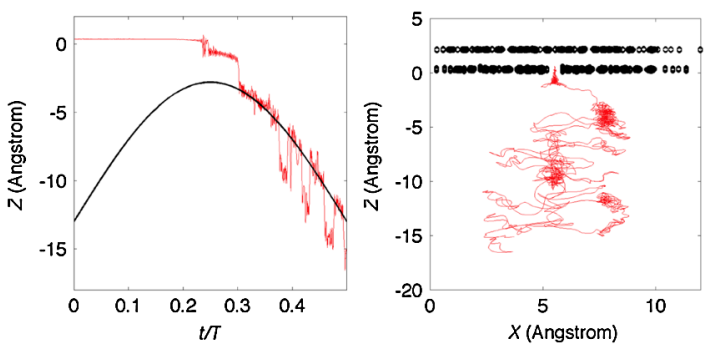

FIG. 2 (color online). Dynamical description of the process of extraction. Left panel: The target atom, shown by a thin (red) oscillating line gets extracted from the lattice under the approach of the AFM tip atom, shown as a thick smooth black line. The time scale is shown in units of the period $T$ of the AFM oscillation. Only the first half of the AFM oscillation is shown, as it demonstrates the approach to the lattice. The second half of the AFM oscillation plays no role in the dynamics. The target atom follows the tip, escaping the bonds with the lattice. Right panel: The motion of lattice atoms, shown as black circles, and the meandering trajectory of target atom, shown as the thin (red) line, as seen from the side. Coordinate $X$ is along the surface, and $Z$ is normal to the surface. 

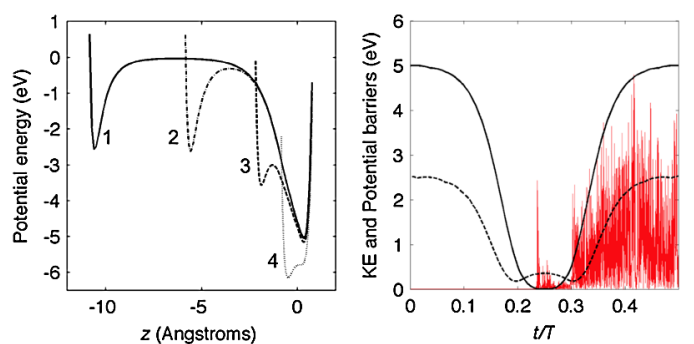

FIG. 3 (color online). Energy balance in the extraction simulation. Left panel: The potential energy profile as a function of normal distance $Z$ from the lattice for different separations $d$ of the tip apex from the surface. 1 (solid black line): $d=13 \AA$; 2 (long dashed line): $d=8 \AA$; 3 (short dashed line): $d=4 \AA$; 4 (dotted line): $d=3 \AA$. Right panel: The time evolution of potential barriers for the target atom and kinetic energy (KE) of the target atom. Solid black line: potential barrier value for the lattice well. Dashed line: potential barrier for the tip well. Kinetic energy is shown as an oscillating thin (red) line. All energy values are in electron Volts $(\mathrm{eV})$.

trated on Fig. 4. All the notation is identical to Fig. 2 for the simplicity of reading. The dynamics of deposition is rather different from that of the extraction due to different values of the potential well in the double-well potential as shown on Fig. 3, left panel. The potential well at the tip is much shallower than then potential well at the surface. Once the atom at the tip acquires sufficient energy to escape from the potential minimum at the tip, that atom will necessarily go directly to the deeper potential minimum at the surface. Thus, the escape from the tip occurs almost instantaneously after the target atom's kinetic energy reaches the value of the potential barrier, as shown on Fig. 3, right panel. Correspondingly, since the total energy of the target atom is small during deposition, the subsequent oscillations of the target atom are rather small, as can be seen by comparing the right part of Figs. 3 and 2. Our results illuminate the nature of asymmetry between the extraction
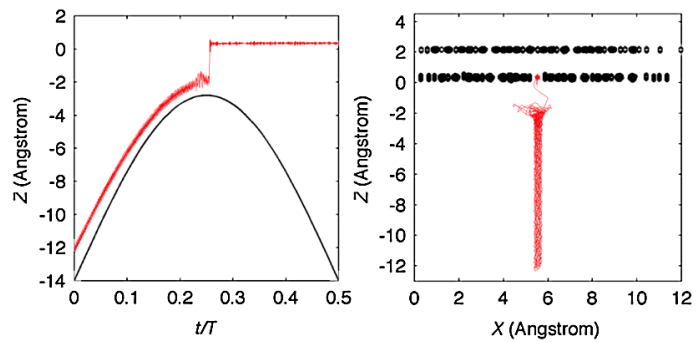

FIG. 4 (color online). Dynamical description of the process of deposition. Left panel: The target atom (thin red line) follows the AFM tip (thick black line) until it approaches the lattice, positioned at $Z=0.2 \AA$. Time is scaled by the period of AFM oscillation $T$. The increase in kinetic energy of the target atom due to the interaction with the lattice atoms allows it to escape the potential well of the tip and bond to the lattice. Right panel: The motion of lattice atoms, shown as black circles, and the target atom, shown in the thin (red) line, as seen from the side. Compare to the corresponding dynamics of extraction as shown on Fig. 2. and deposition as a consequence of a complex interplay between forces of interactions of individual atoms, atomic vibrations, and resonance.

In conclusion, we propose a new explanation of extraction and deposition of atoms using AFM. Our explanation is built on the idea of parametric resonance between the vibrating atoms of the AFM tip and the target atom. In spite of the simplified nature of our models, such as absence of valence bonds, shorter time scales, and a rather idealistic view of the AFM tip, our results demonstrate the importance of atomic vibrations for the explanation of atomic extraction and deposition. As far as we know, this physical effect has not been considered in previous literature based on either quantum or classical mechanical theories. We thus believe that dynamical effects such as atomic vibrations and resonances are very important for an accurate theoretical explanation of atomic manipulations.

B. K. and V.P. were partially supported by Grant No. NSF-DMS-05377891. T.H. was partially supported by 21st Century COE (Program No. 14213201) and Global COE Programs in Kyoto University, funded by MEXT, Japan. In addition, all authors acknowledge the support by Kansai Electric Power Co., Ltd. Japan.

*kimb@math.colostate.edu

[1] Q. Tang, S.-Q. Shi, and L. Zhou, J. Nanosci. Nanotechnol. 4, 948 (2004).

[2] D. Eigler and E. Schwizer, Nature (London) 344, 524 (1990).

[3] L. Bartels, G. Meyer, and K. Rieder, Phys. Rev. Lett. 79, 697 (1997).

[4] J. Stroscio and R. Celotta, Science 306, 242 (2004).

[5] S.-W. Hla, J. Vac. Sci. Technol. B 23, 1351 (2005).

[6] Y.Sugimoto et al., Nature (London) 446, 64 (2007).

[7] N. Oyabu et al., Phys. Rev. Lett. 96, 106101 (2006).

[8] N. Oyabu, O. Custance, M. Abe, and S. Morita, e-J. Surf. Sci. Nanotech. 4, 1 (2006).

[9] N. Oyabu et al., Phys. Rev. Lett. 90, 176102 (2003).

[10] Y. Sugimoto, Ó. O. Custance, M. Abe, and S. Morita, e-J. Surf. Sci. Nanotech. 4, 376 (2006).

[11] Y. Sugimoto et al., Science 322, 413 (2008).

[12] T. Trevethan, M. Watkins, L. Kantorovich, and A. Shluger, Phys. Rev. Lett. 98, 028101 (2007).

[13] P. Dieška, I. Štich, and R. Pérez, Phys. Rev. Lett. 95, 126103 (2005).

[14] P. Dieška and I. Štich, Nanotechnology 18, 084016 (2007).

[15] L. Zhiheng et al., Science 312, 1024 (2006).

[16] H. Goldstein, Classical Mechanics (Addison-Wesley, Reading, Massachusetts, 2001), 3rd ed..

[17] M. Moreno-Moreno, A. Raman, J. Gomez-Herrero, and R. Reifenberger, Appl. Phys. Lett. 88, 193108 (2006).

[18] H. Balamane, T. Halicioglu, and W. A. Tiller, Phys. Rev. B 46, 2250 (1992).

[19] K.-H. Chung, Y.-H. Lee, and D. E. Kim, Ultramicroscopy 102, 161 (2005).

[20] S. Morita et al., e-J. Surf. Sci. Nanotech. 1, 158 (2003).

[21] G. E. Franklin et al., Phys. Rev. B 50, 7483 (1994). 